\title{
Ferric Hydrogensulfate Catalyzed Synthesis of Aryl 14H-Dibenzo[a,j]xanthene Derivatives under Thermal and Solvent-Free Conditions
}

\author{
Hamid Reza Shaterian* and Majid Ghashang
}

Department of Chemistry, Faculty of Sciences, University of Sistan and Baluchestan, PO Box 98135-674, Zahedan, Iran

\begin{abstract}
O hidrogenossulfato férrico tem sido utilizado como catalisador para a preparação em um único pote dos derivados de aril $14 H$-dibenzo[a,j]xantenos por ciclocondensação de $\beta$-naftol e benzaldeídos substituídos, em condições livres de solvente e aquecimento. Este método tem a vantagem de fornecer rendimentos elevados, reações mais limpas, metodologia simples, menor tempo de reação, fácil tratamento e condições experimentais menos poluentes.
\end{abstract}

Ferric hydrogensulfate as catalyst has been used for the one-pot preparation of aryl $14 \mathrm{H}$ dibenzo[a,j]xanthene derivatives by cyclocondensation of $\beta$-naphthol and substituted benzaldehydes under solvent-free and thermal conditions. This method has the advantages of high yields, a cleaner reaction, simple methodology, short reaction times, easy workup and greener conditions.

Keywords: dibenzoxanthene, ferric hydrogensulfate, $\beta$-naphthol, aldehyde, solvent-free

\section{Introduction}

Organic syntheses involving greener process and under solvent-free conditions have been investigated world wide due to stringent environment and economic regulations. ${ }^{1}$ Toxic, homogeneous, corrosive liquid acid catalysts, such as $\mathrm{H}_{2} \mathrm{SO}_{4}, \mathrm{HF}, \mathrm{HBr}, \mathrm{HCl}, \mathrm{CF}_{3} \mathrm{COOH}$ and complexes of $\mathrm{BF}_{3}$ are frequently used in organic synthesis. ${ }^{2}$ However, processes involving conventional acids are inherently associated with problems such as polluting reagents, catalyst waste, difficulty in separation and recovery. ${ }^{3}$ Replacement of these conventional acids by solid heterogeneous catalyst is desirable to achieve effective catalyst handling, product purification and to decrease waste production. ${ }^{4}$ Considering the facts that most of the organic reagents involved in fine chemical synthesis are sensitive to harsh conditions, it is desirable to choose catalysts which can catalyze organic transformations under mild conditions. ${ }^{3,4}$ In this research, ferric hydrogensulfate as the solid heterogeneous acid catalyst deserves special mention. The catalyst has shown strong surface acidic sites, easily recoverable and reusable properties with low toxicity. Also, this catalyst is safe, easy to handle, environmentally benign, presents fewer disposal problems and is stable in reaction media. Ferric hydrogensulfate has been demonstrated to be efficient

*e-mail: hrshaterian@hamoon.usb.ac.ir catalyst for several industrially important reactions such as Schmidt reaction of ketones to amides ${ }^{5}$ and gained much interest in the Friedel-Crafts acylation of alkoxy benzenes by aliphatic anhydrides. ${ }^{6}$

Xanthenes synthesis, especially benzoxanthenes, has attracted the attention of organic chemists due to their wide range of biological and therapeutic properties such as antibacterial, ${ }^{7}$ antiviral activities, ${ }^{8}$ and also they are candidates in photodynamic therapy (PDT). ${ }^{9}, 10$ Furthermore, benzoxanthenes are used as dyes, ${ }^{11}$ in laser technologies, ${ }^{12}$ and in fluorescent materials. ${ }^{13}$

The synthesis of $14 H$-dibenzo[a,j]xanthene is generally achieved by a) dehydration of bis(2-hydroxy-1-naphthyl) methane using $\mathrm{POCl}_{3}{ }^{14}$ or by boiling acetic acid diester of bis(2-hydroxy-1-naphthyl) methane, ${ }^{15} \mathrm{~b}$ ) condensation of $\beta$-naphthol with aliphatic and aromatic aldehydes in the presence of hydrochloric acid or phosphoric acid ${ }^{16}$ and also sulfuric acid ${ }^{17}$ in acetic acid as solvent. All these methods suffer from harsh reaction conditions, long reaction times, unsatisfactory yields and tedious experimental procedures. Recently, the synthesis of $14 H$-dibenzo[a,j]xanthene has been reported by condensation of $\beta$-naphthol and aldehydes in the presence of $p$-toluenesulfonic acid, ${ }^{18,19}$ Amberlyst- $15,{ }^{20}$ molecular iodine, ${ }^{21,22,23}$ sulfamic acid, ${ }^{24}$ silica sulfuric acid, ${ }^{25,26}$ $\mathrm{Al}\left(\mathrm{HSO}_{4}\right)_{3},{ }^{27}$ heteropolyacid, ${ }^{28,29} \mathrm{LiBr}^{30}$ selectfluor $^{\mathrm{TM}},{ }^{31}$ cation-exchange resins, ${ }^{32}$ wet cyanuric chloride, ${ }^{33}$ $\mathrm{HClO}_{4}-\mathrm{SiO}_{2},{ }^{34} \mathrm{~K}_{5} \mathrm{CoW}_{12} \mathrm{O}_{40} \cdot 3 \mathrm{H}_{2} \mathrm{O},{ }^{35}$ methanesulfonic 
acid, ${ }^{36},{ }^{37}$ and dowex $-50 \mathrm{w}^{38}$ as catalyst. Now, we have developed new one-pot methodologies to perform synthesis of aryl $14 H$-dibenzo[a,j]xanthene derivatives by the condensation reaction of various aromatic aldehydes with $\beta$-naphthol in solvent-free condition in the presence of ferric hydrogensulfate under thermal conditions.

\section{Results and Discussion}

Ferric hydrogensulfate as a recyclable solid Brønsted acid catalyst is safe, easy to handle, environmentally benign and presents fewer disposal problems. This catalyst was prepared from the reaction of anhydrous ferric chloride (1 mmol) with concentrated sulfuric acid $(3 \mathrm{mmol})$. This salt is a stable and non-hygroscopic solid material, insoluble in most organic solvents.

In the present research, we wish to describe a new protocol for the rapid preparation of $14 H$-dibenzo[a,j] xanthene derivatives using a catalytic amount of $\mathrm{Fe}\left(\mathrm{HSO}_{4}\right)_{3}$ as a solid acid catalyst (Scheme 1).
As summarized in Table 1, several different aromatic aldehydes and $\beta$-naphthol were reacted in the presence of ferric hydrogensulfate as catalyst under thermal and solvent-free conditions resulting into the corresponding aryl $14 H$-dibenzo[a,j]xanthenes in good to high yields (Scheme 1 and Table 1).

The reaction was performed with benzaldehyde containing withdrawing as well as electron donating groups, but benzaldehydes with electron donating groups are generally more reactive than their corresponding ones with electron withdrawing groups and give the desired product at short reaction time with excellent yield (Table 1). This observation shows clearly that the preparation of benzoxanthenes is more strongly affected by the electronic factors.

The suggested mechanism of the ferric hydrogensulfate catalyzed transformation is shown in Scheme 2.

In a typical experiment, after the necessary time for the reaction to be complete, chloroform was poured in cooled reaction mixtures until solid crude product was dissolved.

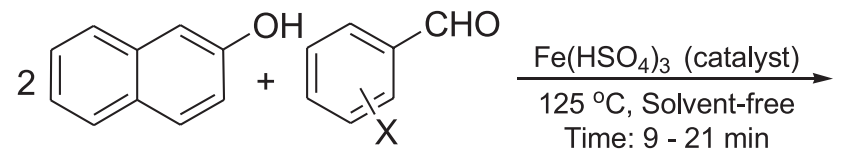

$\mathrm{X}=\mathrm{H}, \mathrm{Cl}, \mathrm{NO}_{2}, \mathrm{CH}_{3}, \mathrm{OH}, \mathrm{OCH}_{3}$

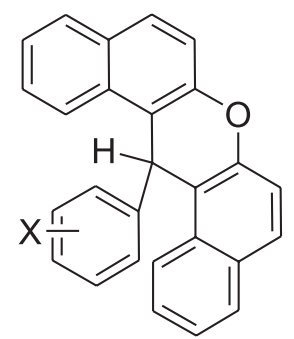

Scheme 1. One-pot preparation of aryl $14 H$-dibenzo[a,j]xanthene derivatives by condensation of $\beta$-naphthol and substituted benzaldehydes in the presence of $\mathrm{Fe}\left(\mathrm{HSO}_{4}\right)_{3}$.

Table 1. Synthesis of aryl $14 H$-dibenzo[a,j]xanthene derivatives in presence of $\mathrm{Fe}\left(\mathrm{HSO}_{4}\right)_{3}$ as catalyst from $\beta$-naphthol and aromatic aldehydes under thermal $\left(125^{\circ} \mathrm{C}\right)$ and solvent-free conditions

\begin{tabular}{|c|c|c|c|c|c|}
\hline Entry & Aldehydes & $\begin{array}{l}\text { time/ } \\
\text { (min) }\end{array}$ & $\begin{array}{l}\text { Yield/ } \\
(\%)\end{array}$ & $\begin{array}{l}\mathrm{mp} /\left({ }^{\circ} \mathrm{C}\right) \\
{[\text { Lit. mp] }}\end{array}$ & $\begin{array}{l}\text { References to the } \\
\text { known product }\end{array}$ \\
\hline 1 & Benzaldehyde & 14 & 97 & $181(183)$ & $17-24$ \\
\hline 2 & 2,4-Dichlorobenzaldehyde & 12 & 91 & 227 & 23,24 \\
\hline 3 & 4-Chlorobenzaldehyde & 12 & 93 & $287(287)$ & $19-24$ \\
\hline 4 & 3-Chlorobenzaldehyde & 13 & 94 & 173 & 23,24 \\
\hline 5 & 4-Nitrobenzaldehyde & 21 & 91 & $315(312)$ & $17,19,21-24$ \\
\hline 6 & 3-Nitrobenzaldehyde & 20 & 96 & $214(213)$ & $19,21-24$ \\
\hline 7 & 4-hydroxybenzaldehyde & 11 & 85 & 111 & 23,24 \\
\hline 8 & 4-Hydroxy-2-methoxybenzaldehyde & 9 & 89 & 131 & 23,24 \\
\hline 9 & 4-Methylbenzaldehyde & 14 & 96 & $227(228)$ & $17-24$ \\
\hline 10 & 2,5-Dimethoxybenzaldehyde & 12 & 87 & 169 & 24 \\
\hline
\end{tabular}

aields refer to the pure isolated products. All known products have been reported previously in the literature and were characterized by comparison of IR and NMR spectra with authentic samples. ${ }^{17-24}$ 


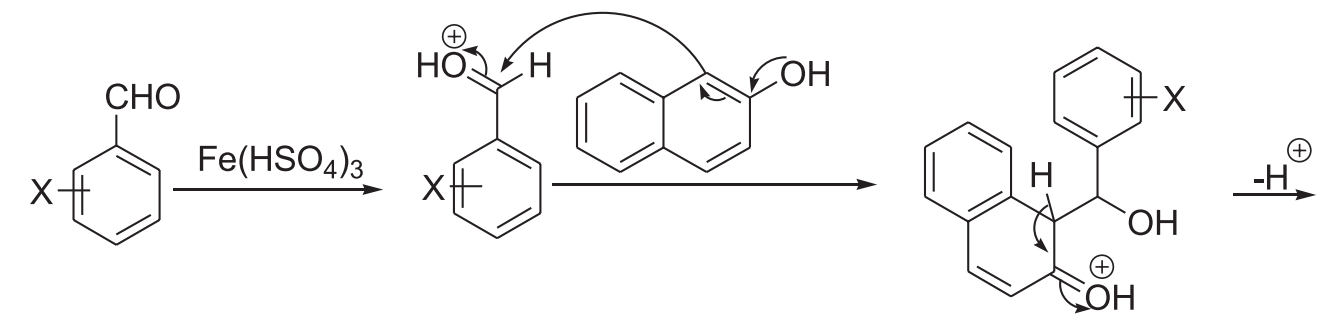

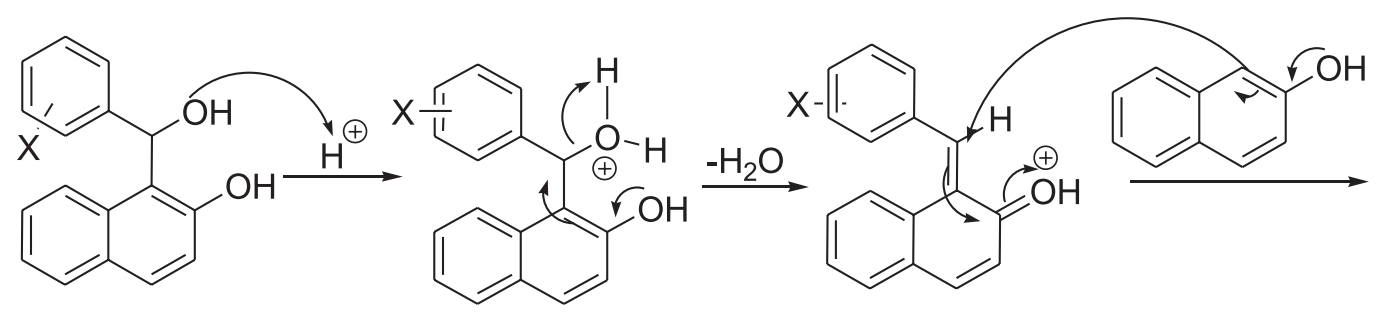<smiles>[Y]c1ccc(C(c2c(O)ccc3ccccc23)C2(C(=O)O)C=Cc3ccccc32)cc1</smiles><smiles></smiles><smiles>[Y4]c1ccc(C2c3c(ccc4ccccc34)OC3(O)C=Cc4ccccc4C23C)cc1</smiles><smiles>[Y]c1ccc(C2c3c(ccc4ccccc34)Oc3ccc4ccccc4c32)cc1</smiles>

Scheme 2. Proposed mechanism for the preparation of 14-aryl-14H-benzo[a,j]xanthene.

Then, the ferric hydrogensulfate as catalyst was isolated from the reaction mixture by simple filtration and could be reused again after washing by chloroform. In continuation of work up, the chloroform was evaporated and the aqueous ethanol $15 \%$ was added to the crude product, the precipitate was separated and then recrystallized using aqueous ethanol $15 \%$ for two times. The catalytic activity of the recovered catalyst, ferric hydrogensulfate, was examined again, and it showed almost the same catalytic activity as fresh ferric hydrogensulfate.

To show the merit of the present work in comparison with reported results in the literature, we compared results of ferric hydrogensulfate with $p$-toluenesulfonic acid, ${ }^{19}$ iodine $^{21}$ and sulfamic acid ${ }^{22}$ in the synthesis of aryl $14 H$-dibenzo[a,j]xanthene derivatives. As shown in Table 2 , ferric hydrogensulfate can act as effective catalyst with respect to reaction times, yields and the obtained products (Table 2).

\section{Conclusions}

In conclusion, we have demonstrated that ferric hydrogensulfate is a new, efficient and heterogeneous catalyst for synthesis of a variety of aryl $14 H$-dibenzo[a,j] xanthene derivatives using aryl aldehydes and $\beta$-naphthol under solvent-free conditions. The reactions were carried out at oil bath with short reaction time and produce the corresponding product in good to high yields. This method is important from an environmental point of view and economic considerations, because it produces little waste. Greener conditions and simple work-up procedure, including filtering and recrystallization are another advantage of this method. 
Table 2. Comparison results of ferric hydrogensulfate with iodine,$^{21}$ sulfamic acid ${ }^{22}$ and $p$-toluenesulfonic acid ${ }^{19}$ in the synthesis of dibenzoxanthene

\begin{tabular}{|c|c|c|c|c|c|}
\hline Entry & Substrate & Catalyst & Substrate/ $\beta$-naphthol/Catalyst & Condition & Yield $/(\%)$ \\
\hline \multirow{4}{*}{1} & & Iodine & $1 / 2 / 0.2$ & Solvent-free; heat $\left(90^{\circ} \mathrm{C}\right)$; time: $2.5 \mathrm{~h}$ & 90 \\
\hline & & sulfamic acid & $1 / 2 / 0.1$ & Solvent-free; heat $\left(125^{\circ} \mathrm{C}\right)$; time: $8.0 \mathrm{~h}$ & 93 \\
\hline & & $p$-toluenesulfonic acid & $1 / 2 / 0.02$ & Solvent-free, heat $\left(125^{\circ} \mathrm{C}\right)$, time: $4.0 \mathrm{~h}$ & 89 \\
\hline & & Ferric hydrogensulfate & $1 / 2 / 0.15$ & Solvent-free, heat $\left(125^{\circ} \mathrm{C}\right)$, time: $14.0 \mathrm{~min}$ & 97 \\
\hline \multirow{4}{*}{2} & & Iodine & $1 / 2 / 0.2$ & Solvent-free; heat $\left(90^{\circ} \mathrm{C}\right)$; time: $2.5 \mathrm{~h}$ & 85 \\
\hline & & sulfamic acid & $1 / 2 / 0.1$ & Solvent-free; heat $\left(125^{\circ} \mathrm{C}\right)$; time: $11.0 \mathrm{~h}$ & 94 \\
\hline & & $p$-toluenesulfonic acid & $1 / 2 / 0.02$ & Solvent-free, heat $\left(125^{\circ} \mathrm{C}\right)$, time: $2.5 \mathrm{~h}$ & 90 \\
\hline & & Ferric hydrogensulfate & $1 / 2 / 0.15$ & Solvent-free, heat $\left(125^{\circ} \mathrm{C}\right)$, time: $21.0 \mathrm{~min}$ & 91 \\
\hline \multirow{4}{*}{3} & & Iodine & $1 / 2 / 0.2$ & Solvent-free; heat $\left(90^{\circ} \mathrm{C}\right)$; time: $3.0 \mathrm{~h}$ & 88 \\
\hline & & sulfamic acid & $1 / 2 / 0.1$ & Solvent-free; heat $\left(125^{\circ} \mathrm{C}\right)$; time: $11.0 \mathrm{~h}$ & 92 \\
\hline & & $p$-toluenesulfonic acid & $1 / 2 / 0.02$ & Solvent-free, heat $\left(125^{\circ} \mathrm{C}\right)$, time: $3.0 \mathrm{~h}$ & 92 \\
\hline & & Ferric hydrogensulfate & $1 / 2 / 0.15$ & Solvent-free, heat $\left(125^{\circ} \mathrm{C}\right)$, time: $14.0 \mathrm{~min}$ & 96 \\
\hline
\end{tabular}

\section{Experimental}

All reagents were purchased from Merck and Aldrich and used without further purification. Ferric hydrogensulfate was prepared according to the reported procedure. ${ }^{5,6}$ All yields refer to isolated products after purification. Products were characterized by comparison with authentic samples and by spectroscopic data (IR, ${ }^{1} \mathrm{H}$ NMR spectra) and melting point. The NMR spectra were recorded on a Bruker Avance DEX 500 and $300 \mathrm{MHz}$ instrument. The spectra were measured in $\mathrm{CDCl}_{3}$ relative to TMS (0.00 ppm). IR spectra were recorded on a Perkin-Elmer 781 spectrophotometer. All of the compounds were solid and solid state IR spectra were recorded using the $\mathrm{KBr}$ disk technique. Melting points were determined in open capillaries with a BUCHI 510 melting point apparatus and are uncorrected. TLC was performed on Silica-gel polygram SIL G/UV 254 plates.

Preparation of $14 H$-dibenzo[a,j]xanthene derivatives using ferric hydrogensulfate as catalyst

To a mixture of aldehyde $(1 \mathrm{mmol})$ and $\beta$-naphthol ( $2 \mathrm{mmol})$, ferric hydrogensulfate $(0.15 \mathrm{mmol}, 0.05 \mathrm{~g})$ was added and the mixture was inserted in an oil bath and heated at $125{ }^{\circ} \mathrm{C}$ for the appropriate time (Table 1). Completion of the reaction was indicated by TLC. After completion, the reaction mass was cooled to $25^{\circ} \mathrm{C}$, then warm aqueous EtOH (15\%) was added and the mixture stirred for $5 \mathrm{~min}$. The solid residue was isolated and dissolved in $\mathrm{CHCl}_{3}$. The ferric hydrogensulfate was filtered; chloroform was evaporated from the reaction mixture. The solid product was purified by recrystallization procedure in aqueous
EtOH (15\%). The desired pure products were characterized by comparison of their physical data with those of known benzoxanthenes ${ }^{17-24}$ The spectral data of some representative aryl $14 H$-dibenzo[a,j]xanthenes are given below:

\section{4-Phenyl-14H-dibenzo[a,j]xanthene (Table 1, Entry 1)}

${ }^{1} \mathrm{HNMR}\left(\mathrm{CDCl}_{3}, 500 \mathrm{MHz}\right): \delta 8.40(2 \mathrm{H}, \mathrm{d}, J 8.5 \mathrm{~Hz}), 7.83$ $(2 \mathrm{H}, \mathrm{d}, 7.9 \mathrm{~Hz}), 7.79(2 \mathrm{H}, \mathrm{d}, 8.8 \mathrm{~Hz}), 7.59(2 \mathrm{H}, \mathrm{t}, 7.7 \mathrm{~Hz}), 7.54$ $(2 \mathrm{H}, \mathrm{d}, 7.5 \mathrm{~Hz}), 7.50(2 \mathrm{H}, \mathrm{d}, 8.8 \mathrm{~Hz}), 7.42(2 \mathrm{H}, \mathrm{t}, 7.5 \mathrm{~Hz}), 7.16$ $(2 \mathrm{H}, \mathrm{t}, 7.5 \mathrm{~Hz}), 7.00(1 \mathrm{H}, \mathrm{t}, 7.5 \mathrm{~Hz}), 6.50(1 \mathrm{H}, \mathrm{s}) ;{ }^{13} \mathrm{C} \mathrm{NMR}$ $\left(\mathrm{CDCl}_{3}, 125 \mathrm{MHz}\right): \delta 148.8,145.0,131.5,131.1,128.8,128.5$, 128.2 126.8, 126.5, 126.4, 124.2, 122.7, 118.0, 117.4, 38.1; IR ( $\left.\mathrm{KBr}, \mathrm{cm}^{-1}\right): 3074,3020,2887,1620,1591,1512,1489$, 1457, 1402, 1253, 1079, 1026, 964, 827, 744, 700.

\section{4-(2,4-Dichloropheny)l-14H-dibenzo[a,j]xanthene (Table 1, Entry 2)}

${ }^{1} \mathrm{H} \mathrm{NMR}\left(\mathrm{CDCl}_{3}, 500 \mathrm{MHz}\right): \delta 8.65(2 \mathrm{H}, \mathrm{d}, J 8.5 \mathrm{~Hz})$, $7.83(2 \mathrm{H}, \mathrm{d}, J 7.9 \mathrm{~Hz}), 7.80(2 \mathrm{H}, \mathrm{d}, J 8.8 \mathrm{~Hz}), 7.63(2 \mathrm{H}, \mathrm{t}, J$ $7.5 \mathrm{~Hz}), 7.48(2 \mathrm{H}, \mathrm{d}, J 8.8 \mathrm{~Hz}), 7.45(2 \mathrm{H}, \mathrm{t}, J 7.4 \mathrm{~Hz}), 7.31$ $(1 \mathrm{H}, \mathrm{d}, J 8.6 \mathrm{~Hz}), 7.27(1 \mathrm{H}, \mathrm{s}), 6.88(1 \mathrm{H}, \mathrm{d}, J 6.8 \mathrm{~Hz}), 6.75$ $(1 \mathrm{H}, \mathrm{s}) ;{ }^{13} \mathrm{C} \mathrm{NMR}\left(\mathrm{CDCl}_{3}, 125 \mathrm{MHz}\right): \delta 148.9,142.2,132.8$, 132.6, 131.6, 130.9, 130.6, 129.3, 129.1, 128.7, 128.4, 127.0, 124.5, 123.1, 118.0, 117.4, 34.3; IR ( $\left.\mathrm{KBr}, \mathrm{cm}^{-1}\right): 3057,2920$, 1619, 1592, 1558, 1514, 1458, 1404, 1240, 1208, 1141, 1101, $1041,960,863,836,808,742,699,607$.

14-(3-Chlorophenyl)-14H-dibenzo[a,j]xanthene (Table 1, Entry 4)

${ }^{1} \mathrm{H}$ NMR $\left(\mathrm{CDCl}_{3}, 300 \mathrm{MHz}\right): \delta 8.33(2 \mathrm{H}, \mathrm{d}, J 8.4 \mathrm{~Hz})$, 
$7.84(2 \mathrm{H}, \mathrm{d}, J 8.6 \mathrm{~Hz}), 7.80(2 \mathrm{H}, \mathrm{d}, J 9.0 \mathrm{~Hz}), 7.61(2 \mathrm{H}, \mathrm{t}, J$ $7.0 \mathrm{~Hz}), 7.49(2 \mathrm{H}, \mathrm{d}, J 8.9 \mathrm{~Hz}), 7.47-7.41(4 \mathrm{H}, \mathrm{m}), 7.09(1 \mathrm{H}$, t, $J 8.0 \mathrm{~Hz}), 6.98(1 \mathrm{H}, \mathrm{d}, J 8.7 \mathrm{~Hz}), 6.45(1 \mathrm{H}, \mathrm{s}) ;{ }^{13} \mathrm{C} \mathrm{NMR}$ $\left(\mathrm{CDCl}_{3}, 75 \mathrm{MHz}\right): \delta 148.7,146.9,134.4,131.2,131.0,129.6$, 129.1, 128.9, 128.3, 126.9, 126.7, 126.4, 124.4, 122.4, 118.0, 116.5, 37.7 ppm; IR (KBr, cm $\left.{ }^{-1}\right): 2922,1621,1590,1509$, 1456, 1431, 1397, 1245, 1065, 959, 812, 775, 746, 691.

14-(4-Nitrophenyl)-14H-dibenzo[a,j]xanthene (Table 1, Entry 5)

${ }^{1} \mathrm{H} \mathrm{NMR}\left(\mathrm{CDCl}_{3}, 300 \mathrm{MHz}\right): \delta 8.29(2 \mathrm{H}, \mathrm{d}, J 8.4 \mathrm{~Hz})$, $7.99(2 \mathrm{H}, \mathrm{d}, J 8.7 \mathrm{~Hz}), 7.86(2 \mathrm{H}, \mathrm{d}, J 4.1 \mathrm{~Hz}), 7.82(2 \mathrm{H}$, d, J $5.4 \mathrm{~Hz}), 7.67(2 \mathrm{H}, \mathrm{d}, J 8.8 \mathrm{~Hz}), 7.61(2 \mathrm{H}, \mathrm{t}, J 5.6 \mathrm{~Hz})$, $7.51(2 \mathrm{H}, \mathrm{d}, J 8.9 \mathrm{~Hz}), 7.44(2 \mathrm{H}, \mathrm{t}, J 7.9 \mathrm{~Hz}), 6.60(1 \mathrm{H}, \mathrm{s})$; ${ }^{13} \mathrm{C} \mathrm{NMR}\left(\mathrm{CDCl}_{3}, 75 \mathrm{MHz}\right): \delta 152.6,148.1,145.9,135.1$, 130.8, 130.7, 129.6, 128.7, 127.2, 124.7, 123.6, 123.1, 117.7, 116.2, 36.4 ppm; IR (KBr, $\left.\mathrm{cm}^{-1}\right)$ : 3070, 2930, 1621, 1591, 1614, 1457, 1400, 1340, 1200, 1140, 1105, 1013, $964,851,827,808,742,690$.

14-(4-Hydroxy-2-methoxyphenyl)-14H-dibenzo[a,j] xanthene (Table 1, Entry 8)

${ }^{1} \mathrm{H} \mathrm{NMR}\left(\mathrm{CDCl}_{3}, 500 \mathrm{MHz}\right): \delta 8.40(2 \mathrm{H}, \mathrm{d}, J 8.2 \mathrm{~Hz})$, $7.83(2 \mathrm{H}, \mathrm{d}, J 7.8 \mathrm{~Hz}), 7.79(2 \mathrm{H}, \mathrm{d}, J 8.7 \mathrm{~Hz}), 7.58(2 \mathrm{H}, \mathrm{t}$, $J 7.2 \mathrm{~Hz}), 7.48(2 \mathrm{H}, \mathrm{d}, J 8.7 \mathrm{~Hz}), 7.42(2 \mathrm{H}, \mathrm{t}, J 7.2 \mathrm{~Hz})$, $7.15(1 \mathrm{H}, \mathrm{d}, J 8.0 \mathrm{~Hz}), 6.85(1 \mathrm{H}, \mathrm{s}), 6.73(1 \mathrm{H}, \mathrm{d}, J 8.0 \mathrm{~Hz})$, $6.44(1 \mathrm{H}, \mathrm{s}), 5.34(1 \mathrm{H}, \mathrm{s}), 3.65(3 \mathrm{H}, \mathrm{s}) ;{ }^{13} \mathrm{C} \mathrm{NMR}\left(\mathrm{CDCl}_{3}\right.$, $125 \mathrm{MHz}): \delta$ 148.7, 146.7, 144.1, 137.1, 131.4, 131.1, $128.8,128.7,126.7,124.2,122.7,121.0,117.9,117.5$, 113.7,110.7, 55.6, 37.5; IR (KBr, $\left.\mathrm{cm}^{-1}\right): 3477,2963,1591$, 1509, 1458, 1430, 1401, 1240, 1032, 959, 805, 781, 749.

14-(4-Methylphenyl)-14H-dibenzo[a,j]xanthene (Table 1, Entry 9)

${ }^{1} \mathrm{H} \mathrm{NMR}\left(\mathrm{CDCl}_{3}, 300 \mathrm{MHz}\right): \delta 8.39(2 \mathrm{H}, \mathrm{d}, J 8.4 \mathrm{~Hz})$, $7.83(2 \mathrm{H}, \mathrm{d}, 8.9 \mathrm{~Hz}), 7.78(2 \mathrm{H}, \mathrm{d}, 8.9 \mathrm{~Hz}), 7.57(2 \mathrm{H}, \mathrm{t}, 8.4$ $\mathrm{Hz}), 7.48$ (2H, d, 8.8 Hz), 7.43-7.38 (4H, m), 6.95 (2H, d, $7.8 \mathrm{~Hz}), 6.46(1 \mathrm{H}, \mathrm{s}), 2.13(3 \mathrm{H}, \mathrm{s}) ;{ }^{13} \mathrm{C} \mathrm{NMR}\left(\mathrm{CDCl}_{3}, 75\right.$ $\mathrm{MHz}): \delta 147.9,147.8,142.5,135.9,130.8,128.8,128.4$, 127.7, 126.7, 124.3, 123.3, 117.5, 117.4, 117.3, 37.1, 20.2; IR $\left(\mathrm{KBr}, \mathrm{cm}^{-1}\right): 3073,2913,1625,1595,1513,1462,1435$, 1405, 1258, 1123, 1086, 966, 841, 813, 785, 744.

14-(2,5-Dimethoxyphenyl-14H-dibenzo[a,j]xanthene (Table 1, Entry 10)

${ }^{1} \mathrm{H} \mathrm{NMR}\left(\mathrm{CDCl}_{3}, 300 \mathrm{MHz}\right): \delta 8.62(2 \mathrm{H}, \mathrm{d}, J 8.4 \mathrm{~Hz})$, $7.82(2 \mathrm{H}, \mathrm{d}, 8.0 \mathrm{~Hz}), 7.78$ (2H, d, $8.9 \mathrm{~Hz}), 7.59$ (2H, t, 7.3
$\mathrm{Hz}), 7.50(2 \mathrm{H}, \mathrm{d}, 8.8 \mathrm{~Hz}), 7.45(2 \mathrm{H}, \mathrm{t}, 7.4 \mathrm{~Hz}), 6.9(1 \mathrm{H}$, s), $6.84(1 \mathrm{H}, \mathrm{s}), 6.78(1 \mathrm{H}, \mathrm{d}, 9.0 \mathrm{~Hz}), 6.48(1 \mathrm{H}, \mathrm{d}, 6.0 \mathrm{~Hz})$, $4.24(3 \mathrm{H}, \mathrm{s}), 3.47(3 \mathrm{H}, \mathrm{s}) ;{ }^{13} \mathrm{C} \mathrm{NMR}\left(\mathrm{CDCl}_{3}, 75 \mathrm{MHz}\right)$ : $\delta$ 154.1, 148.8, 148.2, 135.6, 132.0, 129.9, 129.7, 128.6, 128.5, 126.7, 124.2, 123.4, 118.3, 117.0, 111.9, 111.3, 56.1, 55.2, 30.5; IR $\left(\mathrm{KBr}, \mathrm{cm}^{-1}\right): 2925,2831,1622,1594,1496$, 1461, 1430, 1407, 1256, 1207, 1173, 1043, 965, 849, 813, $800,746,702$.

\section{Supplementary Information}

Supplementary data for the preparation of ferric hydrogensulfate and its FT-IR spectrum are available free of charge at http://jbcs.sbq.org.br, as PDF file.

\section{Acknowledgment}

We are thankful to the Sistan and Baluchestan University Research Council for the partial support of this research.

\section{References}

1. Corma, A.; Garcia, H.; Chem. Rev. 2003, 103, 4307.

2. Van Leeuwen, P.W.; Homogeneous Catalysis: Understanding the Art, Kluwer academic publishers: Boston, 2004.

3. Clark, J.H.; Rhodes, C.N.; Clean Synthesis Using Porous Inorganic Solid Catalysts and Supported Reagents, Royal Society of Chemistry: Cambridge, 2000.

4. Gerard, V.S.; Notheisz, F.; Heterogeneous Catalysis in Organic Chemistry, Elsevier: San Diego, 2000.

5. Eshghi, H.; J. Chin. Chem. Soc. 2006, 53, 987.

6. Salehi, P.; Khodaei, M.M.; Zolfigol, M.A.; Zeinoldini, S.; Synth. Commun. 2003, 33, 1367.

7. Hideo, T.; Jpn. Tokkyo Koho, Chem. Abstr. 1981, 95, 80922b, JP 56005480.

8. Lambert, R.W.; Martin, J.A.; Merrett, J.H.; Parkes, K.E.B.; Thomas, G.J.; Chem. Abstr. 1997, 126, p212377y.

9. Ion, R.M.; Prog. Catal. 1997, 2, 55.

10. Ion, R.M.; Frackowiak, D.; Planner, A.; Wiktorowicz, K.; Acta Biochim. Pol. 1998, 45, 833.

11. Banerjee, A.; Mukherjee, A.K.; Stain Technol. 1981, 56, 83.

12. Ahmad, M.; King, T.A.; Ko, D.-K.; Cha, B.H.; Lee, J.; J. Phys. D: Appl. Phys. 2002, 35, 1473.

13. Knight, C.G.; Stephens, T.; Biochem. J. 1989, 258, 683.

14. Wolf, W.; Chem. Ber. 1893, 26, 83.

15. Rosebush, I.K.; Das Leder 1955, 6, 58.

16. Khorramabadi-zad, A.; Kazemi, Z.; Amini Rudbari, H.; J. Korean Chem. Soc. 2002, 46, 541.

17. Sarma, R.J.; Baruah, J.B.; Dyes Pigm. 2005, 64, 91.

18. Khorramabadi-zad, A.; Akbari, S.A.; Shiri, A.; Veisi, H.; J. Chem. Res. 2005, 277. 
19. Khosropour, A.R.; Khodaei, M.M.; Moghannian, H.; Synlett 2005, 955.

20. Ko, S.K.; Yao, C.-F.; Tetrahedron Lett. 2006, 47, 8827.

21. Das, B.; Ravikanth, B.; Ramu, R.; Laxminarayana, K.; Rao, B.V.; J. Mol. Catal. A: Chem. 255 2006, 255, 74.

22. Pasha, M.A.; Jayashankara, V.P.; Bioorg. Med. Chem. Lett. 2007, 17, 621

23. Ding, F.Q.; An, L.T.; Zou, J.P.; Chin. J. Chem. 2007, 25, 645.

24. Rajitha, B.; Kumar, B.S.; Reddy, Y.T.; Reddy, P.N.; Sreenivasulu, N.; Tetrahedron Lett. 2005, 46, 8691.

25. Shaterian, H.R.; Ghashang, M.; Hassankhani, A.; Dyes Pigm. 2008, 76, 564

26. Seyyedhamzeh, M.; Mirzaei, P.; Bazgir, A.; Dyes Pigm. 2008, 76,836 .

27. Shaterian, H.R.; Ghashang, M.; Mir, N.; Arkivoc 2007, xv, 1.

28. Amini, M.M.; Seyyedhamzeh, M.; Bazgir, A.; Appl. Catal., A: General 2007, 323, 242.

29. Heravi, M.M.; Bakhtiari, K.; Daroogheha, Z.; Bamoharram, F.F.; J. Mole. Catal. A: Chem. 2007, 273, 99.

30. Saini, A.; Kumar, S.; Sandhu, J.S. Synlett 2006, 1928.
31. Kumar, P.S.; Kumar, B. S.; Rajitha, B.; Reddy, P.N.; Sreenivasulu, N.;Reddy, Y.T.; Arkivoc 2006, xii, 46.

32. Patil, S.B.; Bhat, R.P.; Samant, S.D.; Synth. Commun. 2006, 36,2163

33. Bigdeli, M.A.; Heravi, M.M.; Mahdavinia, G.H.; Catal. Commun. 2007, 8, 1595.

34. Bigdeli, M.A.; Heravi, M.M.; Mahdavinia, G.H.; J. Mole. Catal. A: Chem. 2007, 275, 25.

35. Nagarapu, L.; Kantevari, S.; Mahankhali, V.C.; Apuri, S.;Catal. Commun. 2007, 8, 1173.

36. Bhattacharya, A.K.; Rana, K.C.; Mendeleev Commun. 2007, 17,247

37. Liu, D.; Yu, Y.; Shi, W.Z.; Liu, C.S.; Luo, G.X.; Prep. Biochem. Biotechnol. 2007, 37, 77 .

38. Shakibaei, I.G.; Mirzaei, P.; Bazgir, A.; App. Catal. A: General 2007, 325, 188 .

Received: May 30, 2007

Web Release Date: June 5, 2008 


\section{Ferric Hydrogensulfate Catalyzed Synthesis of Aryl 14H-Dibenzo[a,j]xanthene Derivatives under Thermal and Solvent-Free Conditions}

\section{Hamid Reza Shaterian* and Majid Ghashang}

Department of Chemistry, Faculty of Sciences, University of Sistan and Baluchestan, PO Box 98135-674, Zahedan, Iran

\section{Preparation of ferric hydrogensulfate}

A $50 \mathrm{~mL}$ suction flask was equipped with a dropping funnel. The gas outlet was connected to a vacuum system through an alkaline solution trap. Anhydrous ferric chloride (10 mmol) was charged into the flask and concentrated sulfuric acid 98\% (30 mmol) was added dropwise over a period of $30 \mathrm{~min}$ at room temperature. $\mathrm{HCl}$ evolved immediately. After completion of the addition, the mixture was shaken for $30 \mathrm{~min}$ at $100^{\circ} \mathrm{C}$, while the residual $\mathrm{HCl}$ was eliminated by suction. Finally, a pale-brown solid $\mathrm{Fe}\left(\mathrm{HSO}_{4}\right)_{3}(3.41 \mathrm{~g}, 98 \%)$ was obtained according to the previously reported procedure..$^{5,6}$

\section{FT-IR spectrum of $\mathrm{Fe}\left(\mathrm{HSO}_{4}\right)_{3}$}

The FT-IR spectrum of the catalyst was shown in figure 1 . The catalyst is solid and solid state IR spectrum was recorded using the $\mathrm{KBr}$ disk technique. The spectrum shows a broad $\mathrm{OH}$ stretching absorption around 3500 and $3100 \mathrm{~cm}^{-1}$. For sulfuric acid functional group in $\mathrm{Fe}\left(\mathrm{HSO}_{4}\right)_{3}$, the FT-IR absorption of the $\mathrm{O}=\mathrm{S}=\mathrm{O}$ stretching modes lies in $1140 \mathrm{~cm}^{-1}$, and that of the $\mathrm{S}-\mathrm{O}$ stretching mode lies in $600-700 \mathrm{~cm}^{-1}$.

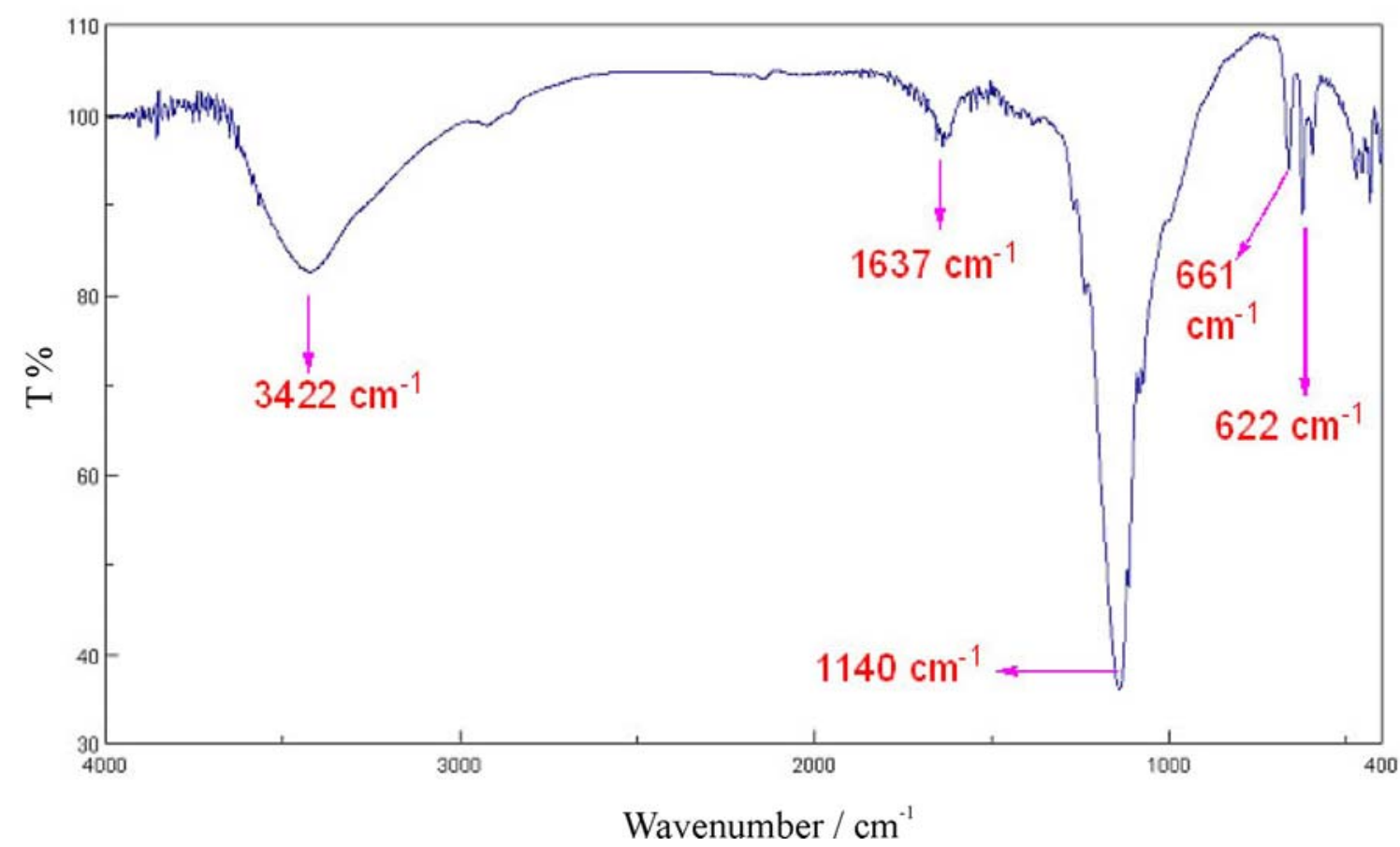

Figure 1. FT-IR spectrum of ferric hydrogensulfate

*e-mail: hrshaterian@hamoon.usb.ac.ir 